\title{
A Path to Qualification of PET/MR Scanners for Multicenter Brain Imaging Studies: Evaluation of MR-based Attenuation Correction Methods Using a Patient Phantom
}

Ciprian Catana ${ }^{1, *}$, Richard Laforest ${ }^{2}, *$, Hongyu $\mathrm{An}^{2}$, Fernando Boada ${ }^{3}$, Tuoyu Cao ${ }^{4}$, David Faul $^{5}$, Bjoern Jakoby ${ }^{6}$, Floris P. Jansen ${ }^{7}$, Bradley J. Kemp ${ }^{8}$, Paul E. Kinahan ${ }^{9}$, Peder Larson ${ }^{10}$, Michael A. Levine ${ }^{1}$, Piotr Maniawski ${ }^{11}$, Osama Mawlawi ${ }^{12}$, Jonathan E. McConathy ${ }^{13}$, Alan B. McMillan $^{14}$, Julie C. Price ${ }^{1}$, Abhejit Rajagopal ${ }^{10}$, John Sunderland ${ }^{15}$, Patrick Veit-Haibach ${ }^{16}$, Kristen A. Wangerin ${ }^{17}$, Chunwei Ying ${ }^{18}$, and Thomas A. Hope ${ }^{10}$

${ }^{1}$ Athinoula A. Martinos Center for Biomedical Imaging, Department of Radiology, Massachusetts General Hospital and Harvard Medical School, Charlestown, MA

${ }^{2}$ Mallinckrodt Institute of Radiology, Washington University School of Medicine, St Louis, MO

${ }^{3}$ Department of Radiology, Center for Advanced Imaging Innovation and Research, New York

University Langone Medical Center, New York, NY

${ }^{4}$ Shanghai United Imaging Healthcare Co., Ltd., Shanghai 201807, China

${ }^{5}$ Siemens Medical Solutions USA, Inc., Malvern, PA, USA

${ }^{6}$ Siemens MR, Siemens Healthcare GmbH, Erlangen, Germany

${ }^{7}$ PET/MR Engineering, GE Healthcare, United States

${ }^{8}$ Division of Nuclear Medicine, Mayo Clinic, Rochester, MN

${ }^{9}$ Imaging Research Laboratory, University of Washington, Seattle, WA

${ }^{10}$ Department of Radiology and Biomedical Imaging, University of California, San Francisco, CA

${ }^{11}$ Philips Healthcare, Advanced Molecular Imaging, Cleveland, OH, USA

${ }^{12}$ Department of Imaging Physics, The University of Texas MD Anderson Cancer Center, Houston, TX

${ }^{13}$ Department of Radiology, University of Alabama at Birmingham, Birmingham, AL

${ }^{14}$ Department of Radiology, University of Wisconsin School of Medicine and Public Health, Madison, WI

${ }^{15}$ Division of Nuclear Medicine, Department of Radiology, University of Iowa, Iowa City, IA 
${ }^{16}$ Toronto Joint Department Medical Imaging, University Health Network, Sinai Health System, Women's College Hospital, Department Medical Imaging University of Toronto, Toronto, Canada

${ }^{17} \mathrm{GE}$ Healthcare, Chicago, IL.

${ }^{18}$ Department of Biomedical Engineering, Washington University in St. Louis, St. Louis, MO

*The authors contributed equally to this work

Running title: PET/MRI scanners trials qualification

\section{Corresponding author:}

Ciprian Catana, $\mathrm{MD}, \mathrm{PhD}$

Athinoula A. Martinos Center for Biomedical Imaging, Department of Radiology, MGH

Bld. $14913^{\text {th }}$ St. Rm. 2.301

Charlestown, MA 02129

Phone: (617) 643-4885

E-mail: ccatana@mgh.harvard.edu

Word count: 5614 


\begin{abstract}
Positron emission tomography and magnetic resonance imaging (PET/MRI) scanners cannot be qualified in the manner adopted for hybrid PET and computed tomography $(\mathrm{CT})$ devices. The main hurdle with qualification in PET/MRI is that attenuation correction (AC) cannot be adequately measured in conventional PET phantoms due to the difficulty in converting the MRI images of the physical structures (e.g., plastic) into electron density maps. Over the last decade, a plethora of novel MR-based algorithms have been developed to more accurately derive the attenuation properties of the human head, including the skull. Although very promising, none of these techniques has yet emerged as an optimal and universally adopted strategy for AC in PET/MRI. In this work, we propose a path for PET/MRI qualification for multicenter brain imaging studies. Specifically, our solution is to separate the head attenuation correction from the other factors that affect PET data quantification and use a patient as a phantom to assess the former. The emission data collected on the integrated PET/MRI scanner to be qualified should be reconstructed using both MR- and CT-based AC methods and whole-brain qualitative and quantitative (both voxelwise and regional) analyses should be performed. The MR-based approach will be considered satisfactory if the PET quantification bias is within the acceptance criteria specified herein. We have implemented this approach successfully across two PET/MRI scanner manufacturers at two sites.
\end{abstract}

Keywords: PET/MRI, attenuation correction, multicenter trials, qualification 


\section{Introduction}

Simultaneous positron emission tomography and magnetic resonance imaging (PET/MRI) scanners were introduced commercially for human imaging in 2010 and have since made their way into research laboratories and clinics following in the footsteps of hybrid PET and computed tomography (PET/CT), which saw its introduction in early 2000. In contrast to PET/CT, which experienced rapid clinical acceptance by providing much needed high-resolution anatomical information and faster attenuation correction (AC) to functional and molecular imaging, combined PET/MRI has seen a much slower acceptance. In addition to the higher cost of the modality, one of the reasons for this slower adoption has been the fact that $\mathrm{AC}$ is more challenging $(1)$ as bone tissue cannot easily be imaged by MR and may be misclassified, resulting in quantitative uncertainties that have helped to perpetuate the viewpoint that PET/MRI remains investigational.

Over the last decade, several MR-based algorithms have been developed to more accurately derive the PET (511 keV) attenuation properties of the human head, including the bone tissue. Algorithms such as UTE (ultra-short time of echo), ZTE (zero time of echo), atlas-based, or most recently, machine learning approaches have been proposed to replace or complement the vendor-provided 2-point DIXON (or LAVA-Flex) sequence that is routinely used in clinical settings (interested readers are referred to $(1,2)$ for recent reviews on this topic). These MR-based attenuation correction (MRAC) algorithms have been evaluated by imaging patients sequentially on PET/CT and PET/MRI scanners and using the CT-based AC (CTAC) as the gold standard. Considering these developments and the need for scanner validation, a clear path to the qualification of this modality is both timely and necessary. Although methods to perform a transmission scan inside the PET/MR scanner have also been proposed (3-5) and could also be used for validating MRbased AC approaches, they require additional hardware and expertise. 
A related limitation to clinical acceptance and inclusion in clinical trials has been that PET/MRI scanners cannot be qualified in the manner adopted for PET/CT. PET/CT qualification or scanner validation, for purposes ranging from clinical use to participation in a clinical trial with PET quantitative endpoints, typically proceeds with scanning standardized phantoms (filled with a radiotracer mixed with water) of pre-defined geometry such as the American College of Radiology (ACR), Clinical Trials Network (CTN) or National Electrical Manufacturers Association International Electrotechnical Commission (NEMA-IEC) phantoms. For PET/CT scanners this works well as the linear attenuation coefficient of water is close to that of soft tissue for both PET and CT. Additionally, CT provides sufficient information to infer the linear attenuation coefficients of other materials (6). These traditional phantoms, however, cannot be imaged accurately by MR because proton properties in magnetic fields do not readily translate to electron density, atomic structure and $511 \mathrm{keV}$ photon attenuation. Specifically, the transverse relaxation time (T2) of protons in phantom materials such as plastic is too short to be captured conventionally, leading to little measurable signal from nearly all types of MR pulse sequences. While substantial progress has been made in manufacturing phantoms capable of mimicking both electron density and MRI contrast characteristics of human tissues (7-9), no such phantom that could be used to assess the performance of multiple MR-based AC techniques is yet widely available. Additionally, water-filled phantoms, a mainstay in the accreditation of PET scanners (10), produce resonance artifacts in MR images (11). In other words, although the standard phantoms accurately replicate the imaging physics of PET and CT for patients, the same is not true for MRI.

In this work, we propose a path for PET/MRI qualification for brain imaging studies using a patient as a phantom. We first explain the differences between accreditation and qualification, 
outline the need for both, then review the accreditation and qualification process in the context of PET/CT, and describe the proposed solution in terms of data acquisition and analysis and the definition of qualification criteria.

\section{Accreditation and Qualification}

The term "accreditation" is used primarily in the clinical setting. For example, all centers in the US that bill for nuclear medicine procedures are required to be accredited to receive all the reimbursement from Medicare. The term "qualification" describes the process of determining if a specific scanner can be used in the setting of a specific clinical trial. Frequently, Contract Research Organizations will require specific phantom imaging tests to qualify scanners prior to allowing sites to enroll in imaging trials. In many settings, approaches to accreditation are used as part of a qualification process. There are many organizations that provide qualification services in the setting of multicenter clinical trials $(12,13)$. Though the terms accreditation and qualification are often used interchangeably, it is important to understand the distinctions between them. The goal of this manuscript is to propose an approach to PET/MRI qualification for brain studies, such that these devices can be utilized for multi-center clinical trials.

\section{Approaches Used in PET/CT}

One of the most commonly used means of accreditation in PET/CT is the ACR accreditation program $(10,14)$. The ACR accreditation program defines the requirements of the personnel performing and interpreting the study, quality control, and peer-review. Additionally, each site must provide images of a specific PET phantom and clinical images that are reviewed centrally. The phantom and clinical images are evaluated qualitatively prior to accreditation. The phantom 
images have specific quantitative acceptance criteria. For example, ACR requires $\pm 15 \%$ error in SUV of the background (as well as other requirements for contrast recovery). Accreditation does not define the performance of the procedure (e.g., uptake time, injected activity, etc.), but rather focuses on the facility, personnel, device, and resultant images. Other organizations also provide accreditation services, such as the Intersocietal Accreditation Commission, RadSite and the Joint Commission $(15,16)$.

For qualification, many clinical trials will accept ACR accreditation, but those focused on novel radiotracers and/or quantitative PET measures frequently require more stringent approaches, which can overlap with harmonization. "Harmonization" is a term that describes setting up the image acquisition and reconstruction parameters so that approximately the same quantitative outcomes are obtained independent of scanners; this approach is sometimes used in trials with quantitative primary and/or secondary endpoints. Two main approaches for harmonization are

those put forth by the CTN and the European Association of Nuclear Medicine Research Ltd (EARL) $(13,17)$. These approaches use phantoms with spheres of varying sizes, filled following exact phantom preparation procedures, to determine harmonized reconstruction parameters capable of producing quantitative results that yield measured standardized uptake values (SUVs) within a predetermined range. Using these approaches, variability in PET quantification can be minimized across imaging devices.

All accreditation, qualification and harmonization procedures in PET/CT require the imaging of a phantom filled with a known quantity of radiotracer in a water solution.

\section{Proposed Solution for PET/MRI scanners: Qualification Using Human Phantoms}


Given the above-mentioned challenges in imaging standard phantoms, a more manageable approach for PET/MRI qualification is to evaluate the PET reconstruction pipeline's constituent parts independently. Specifically, the challenge in the generation of the attenuation map can be isolated from the other effects that affect the PET quantification (i.e., corrections for randoms, dead-time, decay, etc. and those related to the image reconstruction). To address the former challenge, we propose to use patients scanned sequentially on both (PET)/CT and PET/MRI as "phantoms" and evaluate the difference in the resultant attenuation maps and impact on PET data quantification. This approach builds on the methodology typically used for validating MRAC using CTAC as the standard. We propose below procedures to standardize this approach so it can be used to qualify a particular PET/MRI scanner. The guideline recommended here is specific to the head but could in principle be adapted to other more complex regions, although additional challenges would obviously need to be considered for whole-body applications (1). Other factors relevant for the qualification of the PET component of the integrated PET/MRI scanner in a multicenter trial will be assessed using separate imaging phantoms and procedures already in place for PET/CT.

\section{$\underline{\text { Data acquisition }}$}

CT data acquisition: A non-contrast CT study should be performed using parameters typically used for AC in PET scans or diagnostic examinations according to the clinical protocols, and the images should be reconstructed using standard algorithms (e.g., analytical filtered back projection, iterative techniques, etc.). The subject should be positioned on the CT scanner with the arms outside the field of view (i.e., "arms down" as is typically done for head PET/CT and PET/MRI examinations) and the entire head should be scanned (i.e., from top of the head to the lower neck). 
Patients with metallic implants should not be used as they could bias both the CT- and MR-based attenuation maps. Additionally, subjects should be excluded if significant artifacts (e.g., streaks, motion, scanner malfunction, etc.) are seen in the CT images.

MRI data acquisition: MR data should be acquired using the radiofrequency coil that will be used in the clinical study or clinical trial. The site-specific MR sequence(s) used for generating the attenuation map (e.g., Dixon-VIBE, MPRAGE, ZTE, UTE, etc.) should also be acquired with the same parameters as those used in the clinical trial. The whole head (including nose, ears) and the part of the neck present in the physical PET field of view should be covered. If the site-specific MRAC method is different from the vendor-specific one, the vendor-specific MRAC sequences should also be acquired. Additionally, a vendor-specific sequence for obtaining high resolution morphological MRI data (e.g., MPRAGE or BRAVO sequences with approximately $1 \mathrm{~mm}^{3}$ resolution and maximum $1.5 \mathrm{~mm}$ slice thickness) should be acquired for the purposes of image registration to the $\mathrm{CT}$ and region-of-interest (ROI) definition. Any MR images with artifacts that are known to bias the PET data quantification (e.g., susceptibility, water-fat inversion, ghosting, motion) should be excluded from the evaluation. Dental fillings, that might be present in many subjects, are not usually leading to "significant artifacts" and would not be excluded. The MRbased attenuation map should be generated using the site-specific algorithm to be used in the clinical trial (either developed in-house or provided by the manufacturer).

PET data acquisition: The radiotracer used for evaluation will depend on the specific study. The emission data should be acquired using the integrated PET/MRI in one of the following two ways: PET/CT followed by same-day PET/MRI or CT-only followed by same-week PET/MRI. In the 
first scenario, the subject should undergo the additional PET/MRI examination within a reasonable amount of time specific to the radiotracer to provide adequate counts in the PET data acquired on the PET/MRI (e.g., within 3 hours from the time of $\left[{ }^{18} \mathrm{~F}\right] \mathrm{FDG}$ administration). The emission data acquired as part of the PET/CT examination are not used in the analysis, as the focus is on analyzing the impact on the PET data quantification acquired on the PET/MRI scanner. In the second scenario, the PET/MRI examination should be scheduled within one week of the clinical CT scan. As significant changes could occur within a week even without surgical interventions (e.g., differences in the filling of the sinuses could introduce bias in adjacent gray matter structures), subjects with recent onset of upper respiratory infections, acute sinusitis, etc. should be excluded. The acquisition duration on the PET/MRI device should be at least 10 minutes in both scenarios and the emission data should be saved in a manner that permits retrospective reconstruction (i.e., list mode or sinograms, plus associated data for corrections).

In both cases, the patients should be scanned with arms down with the head positioned in the MR scanner as similarly as possible to the CT scan. Specifically, the technologist should review the CT images and try to position the head in a similar orientation with respect to the neck (e.g., no head lateral rotation and similar degree of flexion) to minimize the need for non-rigid body registration. Additionally, the head should be centered in the PET axial field of view to ensure full coverage in a single acquisition.

\section{Centralized Data Processing and Analysis}

We recommend the creation of a PET/MRI scanner accreditation group/organization to perform the steps described below. This group should have the capability to process and analyze 
the data collected on any of the PET/MRI scanners and reproduce all the steps described below using the following data:

- $\quad$ Site-specific MR-based attenuation map;

- $\quad$ Morphological MR images;

- $\quad$ CT images (or CT-based attenuation map);

- Raw emission data in sinogram or list mode format and the additional files required for image reconstruction (e.g., normalization file, hardware attenuation maps).

To minimize the contribution of factors not related to the MR-based attenuation map generation procedure, the accreditation group will use software provided by the manufacturers or freely available packages to standardize the following steps.

\section{$\underline{\text { Data processing }}$}

CT data processing: First, the patient bed and head holder will be removed from the CT images using vendor-provided software. Second, the CT volume will be co-registered to the morphological high-resolution MR volume using rigid body registration with normalized mutual information as the objective function (e.g., using the Elastix $(18,19)$, Statistical Parametric Mapping (20), ITK $(21,22)$ or similar software). The accuracy of the co-registration will be assessed visually by an experienced reader. Third, the Hounsfield units will be converted to linear attenuation coefficients at $511 \mathrm{keV}$ using the vendor-specific procedure. Fourth, the resulting CTbased attenuation maps will be smoothed using a Gaussian filter (with a kernel size that ensures the resulting attenuation maps match the PET scanner spatial resolution) and resampled into the PET space of the specific PET/MRI device. If the CT-based attenuation map is incomplete (due to 
the shorter axial coverage in the neck region or different positioning between the two examinations), the missing data will be copied from the MR-based attenuation map. Finally, the attenuation map will be exported in a format that allows its use for AC using the standard PET image reconstruction pipeline.

PET data processing: The PET images will be reconstructed with the image reconstruction algorithm used in the clinical trial using both the CT- and MRAC maps created above. Typically, the scatter correction provided by the manufacturer will be used in both cases (Note: although the attenuation map is usually used for scatter estimation, only the joint impact of both attenuation and scatter corrections on PET data quantification is of interest here). Post-reconstruction smoothing will be applied according to the study protocol.

\section{$\underline{\text { Data analysis }}$}

ROIs definition: Subject-specific ROIs will be defined from the morphological MR data using FreeSurfer (23). A representative subset of study-specific ROIs will be selected for regional data analysis. Additionally, a brain mask (i.e., all the voxels corresponding to grey and white matter) will be obtained from the MR data.

Quantitative evaluation of the attenuation corrected PET data: The bias present in the PET images reconstructed with MRAC relative to those reconstructed with CTAC will be assessed by computing the voxel-wise percent differences throughout the whole brain mask (i.e., all the voxels corresponding to brain tissue). Additionally, a regional analysis will be performed using the 
FreeSurfer-defined ROIs. Average percent differences as well as average absolute percent differences will be computed for all the selected ROIs.

\section{Qualification criteria}

QC\#1. The MR-based attenuation maps and corresponding PET images should be free of artifacts (e.g., fat/water inversion, susceptibility artifacts in the MR-based map or streak artifacts in the CT-based map, incomplete head coverage, etc.), and no obvious misregistration should be noted in the overlaid images.

QC\#2. The voxel-wise relative differences between the PET images attenuation corrected using the MR- and CT-based approaches should be below 10\% in at least $90 \%$ of the voxels included in the brain mask.

QC\#3. The average absolute percent differences between the PET images attenuation corrected using the MR- and CT-based approaches should be below $10 \%$ in all study-specific ROIs.

QC\#4. For studies involving reference tissue analysis (e.g., standardized uptake value ratios for amyloid PET imaging in neurodegeneration), a more stringent threshold could be set for the reference ROI (e.g., less than 5\% bias in the cerebellum in the case of amyloid PET imaging).

\section{Example Application}

\section{Methods}

The procedures described above were followed for acquiring, processing and analyzing the data to qualify two different PET/MRI scanners for a hypothetical study aimed at assessing $\beta$ amyloid $(A \beta)$ accumulation in Alzheimer's disease subjects. A total of 10 datasets were assessed, 
obtained from 5 subjects scanned on the Siemens Biograph mMR and 5 on the GE Signa PET/MRI scanners. The results from representative cases are discussed below.

At one institution, subjects underwent ${ }^{18} \mathrm{~F}-\mathrm{AV}-45$ (florbetapir) imaging using the GE SIGNA PET/MRI and Discovery STE PET/CT scanners (General Electric Healthcare, Waukesha, WI). The MR-based attenuation maps were generated using atlas- $(24,25)$ and ZTE-based $(26,27)$ approaches. At the other institution, subjects underwent ${ }^{18} \mathrm{~F}-\mathrm{AV}-45$ imaging using the Biograph mMR (Siemens Healthineers, Erlangen, Germany) and Biograph Vision PET/CT scanners (Siemens Healthineers, Knoxville, TN, USA). The MR-based attenuation maps were generated using the Dixon- (28) and skull model-based (29) approaches. The FreeSurfer-derived cortical ROIs were combined into four study-specific large bilateral regions (frontal, cingulate, parietal and lateral temporal) previously proposed for assessing $A \beta$ deposition in this patient population $(30,31)$. Additionally, bilateral regions corresponding to white matter and whole cerebellum were defined.

\section{$\underline{\text { Results }}$}

The attenuation maps and the corresponding PET images for two representative subjects free of artifacts and properly registered are presented in Figure 1 and Supplemental Figures 1 and 2 (QC\#1).

Figure 2 shows the cumulative pixel-wise absolute difference histogram (blue) and pixel-wise absolute percent difference histogram (green) for Dixon- and skull model-based AC on the Siemens Biograph mMR, and atlas- and ZTE-based AC on the GE SIGNA ${ }^{\mathrm{TM}}$ PET/MR scanners. The relative differences between the PET images obtained using the Siemens skull model- and GE ZTE-based methods with respect to the CT-based approach were below $10 \%$ in $94.67 \%$ and 
$96.59 \%$ of the voxels included in the brain mask, respectively (QC\#2). On the other hand, the Siemens Dixon- and GE atlas-based approaches did not meet this acceptance criterion.

The regional absolute relative differences were below $10 \%$ for all the study-specific ROI described above for the Siemens skull model- and GE ZTE-based approaches as depicted in the Bland-Altman plots shown in Figure 3 (QC\#3). The Siemens Dixon- and GE atlas-based approaches did not meet this acceptance criterion.

Plots of cumulative histogram of absolute pixel-wise differences and a summary report for all 10 subjects included in the analysis are given in Figure 4 and Supplemental Table 1. The relative differences between the PET images obtained using the Siemens skull model- and GE ZTE-based methods with respect to the CT-based approach were below $10 \%$ in more than $90 \%$ of the voxels included in the brain mask for all the subjects (QC\#2). On the other hand, the Siemens Dixon- and GE atlas-based approaches did not meet this acceptance criterion for 5 and 3 of the subjects, respectively.

\section{Discussion}

We have proposed to use the patient as a phantom to qualify PET/MRI scanners for brain imaging multicenter trials. Due to the absence of suitable phantoms to evaluate MRAC methods, patient phantoms provide the fastest path forward to evaluating quantitative errors associated with AC. The main advantage of this approach is that it will remain robust independent of the MRAC methodology over time. It is important to note that site qualification for evaluating reconstruction methods is still required; this can be done using standard PET/CT phantoms.

Although a study-specific radiotracer is preferred, ${ }^{18} \mathrm{~F}-\mathrm{FDG}$ may also suffice in many indications due to its global uptake pattern, making the assessment of bias from the MRAC 
generalizable to most other radiotracers relevant to neurological applications. Although the evaluation methods and qualification criteria were defined to ensure the assessment is applicable across radiotracers, additional radiotracer- or patient population-specific assessments could be defined and performed if needed. Finally, the proposed methods are also applicable to AC methods that use the emission data to estimate the attenuation map (32) as well as the latest generation of machine learning approaches (33), including those methods that generate attenuation- and scattercorrected images directly from the non-corrected images without needing to generate an attenuation map (34).

Given the complexity of this method compared to the one used for qualifying PET/CT scanners using innate phantoms, we have recommended that the data processing be performed by the accreditation group/organization. While each site would have to submit images and raw data, this task does not require advanced software or training. The centralized processing would ensure all the steps are performed consistently. The differences between the off-line and online data processing tools could be minimized by obtaining from the equipment manufacturers the tools corresponding to the software version installed on the scanner to be qualified. Furthermore, the PET images attenuation corrected using the CT- and MR-based attenuation maps would be reconstructed using the same input parameters. For these reasons, the remaining differences between the off-line and online reconstructions would not affect the quantitative evaluation of the AC procedure, which is our only goal here. Other effects relevant for PET data quantification would be assessed using images of standard phantoms (e.g., NEMA, SNMMI CTN phantom) reconstructed using the online tools to ensure the images meet the study specific criteria related to image uniformity, spatial resolution, image quality, etc. 
One limitation of the proposed approach is that it requires CT data to be acquired either onsite or at a different facility. Furthermore, the need to perform two examinations places additional burden on the participants, staff and increases the costs compared to scanning an innate phantom. The radiation exposure is also increased in the CT-only followed by PET/MRI examination scenario.

Another drawback to the proposed approach is that each imaging center is required to transfer raw data (i.e., list mode or sinogram PET data) to a central processing site to have the dataset reconstructed using both CT- and MR-based attenuation maps. Sites may not be immediately familiar with how to access and export these large datasets. There is also potential variability associated with CT-to-MR registration. Nonetheless in our initial evaluation of the proposed approach we were able to successfully implement the process across two centers using two different PET/MRI manufacturers, with comparable results. Further work needs to be performed to automate the analysis and to minimize the burden on the central site.

The proposed solution was here applied to the brain, but future work will focus on extending the patient phantom to other parts of the body as accurate MRAC approaches become available. Regional analysis and the impact of MRAC on focal lesion uptake would have to be defined outside of the brain (as well as for assessing the impact of AC in the presence of bone lesions in the head). This translation to other body regions will also be facilitated by using a patient phantom, as new phantom geometries do not need to be developed.

Lastly, this approach uses a best-case scenario patient selected by the individual site, as is done with other qualification approaches, but it does not evaluate the variability across patients. The goal of this qualification approach is to demonstrate that the MRAC methods used on the sitespecific scanner are functioning as expected based upon manufacturer recommendations. As with 
all qualification approaches, this approach does not prevent errors in PET quantitation due to large patient-level abnormalities. It was also not our goal to propose a guideline for harmonization of AC methods. However, the proposed method could be adapted for this purpose although that would require different data acquisition and processing protocols (e.g., scanning the same subject on different PET/MRI scanners).

\section{Conclusion}

We have proposed a solution for qualifying PET/MRI scanners for brain imaging clinical trials. The most significant challenge is to develop PET/MRI specific phantoms that are applicable across different MRAC approaches. To address this issue, we have proposed using the patient as a phantom, whereby the scaled CT attenuation map is used to validate the MR-based map generated for the same patient. The approach was successfully implemented across two PET/MRI scanner manufacturers at two sites.

\section{Disclosures}

Tuoyu Cao is employed by United Imaging Healthcare; David Faul and Bjoern Jakobi are employed by Siemens Healthineers; Floris Jansen and Kristen Wangerin are employed by GE Healthcare; Piotr Maniawski is employed by Philips Healthcare. No other potential conflicts of interest relevant to this article exist. This work was partially supported by the National Institutes of Health (grants 5R01CA212148 and 1U01EB029826). 


\section{KEY POINTS}

QUESTION: Can patient phantoms be used to test the head MR-based attenuation correction methods to qualify PET/MRI scanners for multicenter trials?

PERTINENT FINDINGS: In this consensus paper, we proposed to use a patient as a phantom to assess the accuracy of MR-based attenuation correction using CT as the reference standard. Following the proposed guidelines for data acquisition, image reconstruction and data analysis, we have tested the proposed approach successfully across two PET/MRI scanner manufacturers at two sites.

IMPLICATIONS FOR PATIENT CARE: Integrated PET/MRI scanner can be qualified for multicenter trials focused on neurological applications. 


\section{References}

1. Catana C. Attenuation correction for human PET/MRI studies. Phys Med Biol. 2020;65:23TR02.

2. Chen Y, An H. Attenuation Correction of PET/MR Imaging. Magn Reson Imaging Clin N Am. 2017;25:245-255.

3. Bowen SL, Fuin N, Levine MA, Catana C. Transmission imaging for integrated PET-MR systems. Phys Med Biol. 2016;61:5547-68.

4. Farag A, Thompson RT, Thiessen JD, Prato FS, Théberge J. Improved PET/MRI accuracy by use of static transmission source in empirically derived hardware attenuation correction. EJNMMI Phys. 2021;8:24.

5. Mollet P, Keereman V, Bini J, Izquierdo-Garcia D, Fayad ZA, Vandenberghe S. Improvement of Attenuation Correction in Time-of-Flight PET/MR Imaging with a Positron-Emitting Source. J Nucl Med. 2014;55:329-336.

6. Kinahan PE, Hasegawa BH, Beyer T. X-ray-based attenuation correction for positron emission tomography/computed tomography scanners. Semin Nucl Med. 2003;33:166179.

7. Harries J, Jochimsen TH, Scholz T, et al. A realistic phantom of the human head for PETMRI. EJNMMI Phys. 2020;7:52.

8. Mitsouras D, Lee TC, Liacouras P, et al. Three-dimensional printing of MRI-visible phantoms and MR image-guided therapy simulation. Magn Reson Med. 2017;77:613-622.

9. Rausch I, Valladares A, Sundar LKS, et al. Standard MRI-based attenuation correction for PET/MRI phantoms: a novel concept using MRI-visible polymer. EJNMMI Phys. 2021;8:18.

10. MacFarlane CR. ACR accreditation of nuclear medicine and PET imaging departments. $J$ Nucl Med Technol. 2006;34:18-24.

11. Ziegler S, Braun H, Ritt P, Hocke C, Kuwert T, Quick HH. Systematic Evaluation of Phantom Fluids for Simultaneous PET/MR Hybrid Imaging. J Nucl Med. 2013;54:14641471 .

12. Scheuermann JS, Saffer JR, Karp JS, Levering AM, Siegel BA. Qualification of PET scanners for use in multicenter cancer clinical trials: The American College of Radiology Imaging Network experience. J Nucl Med. 2009;50:1187-1193.

13. Aide N, Lasnon C, Veit-Haibach P, Sera T, Sattler B, Boellaard R. EANM/EARL harmonization strategies in PET quantification: from daily practice to multicentre oncological studies. Eur J Nucl Med Mol Imaging. 2017;44:17-31. 
14. Complete Accreditation Information: Nuclear Medicine and PET (Revised 12-12-19) : Accreditation Support.

https://accreditationsupport.acr.org/support/solutions/articles/11000063279-completeaccreditation-information-nuclear-medicine-and-pet.

15. Accreditation Commission I. The IAC Standards and Guidelines for Nuclear/PET Accreditation.

16. Accreditation Handbook for Diagnostic Imaging Centers.

17. Sunderland JJ, Christian PE. Quantitative PET/CT scanner performance characterization

based upon the society of nuclear medicine and molecular imaging clinical trials network oncology clinical simulator phantom. J Nucl Med. 2015;56:145-152.

18. Klein S, Staring M, Murphy K, Viergever MA, Pluim JPW. elastix: A Toolbox for Intensity-Based Medical Image Registration. IEEE Trans Med Imaging. 2010;29:196-205.

19. Shamonin D, Bron E, Lelieveldt B, Smits M, Klein S, Staring M. Fast Parallel Image Registration on CPU and GPU for Diagnostic Classification of Alzheimer's Disease. Front Neuroinform. 2014;7:50.

20. Penny WD, Friston KJ, Ashburner JT, Kiebel SJ, Nichols TE. Statistical parametric mapping: the analysis of functional brain images. Elsevier; 2011.

21. Yoo TS, Ackerman MJ, Lorensen WE, et al. Engineering and algorithm design for an image processing Api: a technical report on ITK--the Insight Toolkit. Stud Health Technol Inform. 2002;85:586-592.

22. McCormick M, Liu X, Ibanez L, Jomier J, Marion C. ITK: enabling reproducible research and open science. Front Neuroinform. 2014;8:13.

23. Fischl B. FreeSurfer. Neuroimage. 2012;62:774-781.

24. Wollenweber SD, Ambwani S, Delso G, et al. Evaluation of an Atlas-Based PET Head Attenuation Correction Using PET/CT \&amp; MR Patient Data. IEEE Trans Nucl Sci. 2013;60:3383-3390.

25. Sekine T, Buck A, Delso G, et al. Evaluation of Atlas-Based Attenuation Correction for Integrated PET/MR in Human Brain: Application of a Head Atlas and Comparison to True CT-Based Attenuation Correction. J Nucl Med. 2016;57:215-220.

26. Wiesinger F, Sacolick LI, Menini A, et al. Zero TE MR Bone Imaging in the Head. Magn Reson Med. 2016;75:107-114.

27. Sekine T, Ter Voert EEGW, Warnock G, et al. Clinical evaluation of zero-echo-time attenuation correction for brain 18F-FDG PET/MRI: Comparison with atlas attenuation correction. J Nucl Med. 2016;57:1927-1932. 
28. Delso G, Martinez-Moller A, Bundschuh RA, Nekolla SG, Ziegler SI. The effect of limited MR field of view in MR/PET attenuation correction. Med Phys. 2010;37:28042812.

29. Paulus DH, Quick HH, Geppert C, et al. Whole-Body PET/MR Imaging: Quantitative Evaluation of a Novel Model-Based MR Attenuation Correction Method Including Bone. J Nucl Med. 2015;56:1061-6.

30. Mormino EC, Betensky RA, Hedden T, et al. Amyloid and APOE $\varepsilon 4$ interact to influence short-term decline in preclinical Alzheimer disease. Neurology. 2014;82:1760-1767.

31. Buckley RF, Mormino EC, Rabin JS, et al. Sex Differences in the Association of Global Amyloid and Regional Tau Deposition Measured by Positron Emission Tomography in Clinically Normal Older Adults. JAMA Neurol. 2019;76:542-551.

32. Rezaei A, Defrise M, Bal G, et al. Simultaneous Reconstruction of Activity and Attenuation in Time-of-Flight PET. Med Imaging, IEEE Trans. 2012;31:2224-2233.

33. Liu F, Jang H, Kijowski R, Bradshaw T, McMillan AB. Deep learning MR imaging-based attenuation correction for PET/MR imaging. Radiology. 2018;286:676-684.

34. Yang J, Park D, Sohn JH, Wang ZJ, Gullberg GT, Seo Y. Joint Correction of Attenuation and Scatter Using Deep Convolutional Neural Networks (DCNN) for Time-of-Flight PET. Phys Med Biol. 2019;64:16-19. 
Figure 1: Attenuation maps and corresponding ${ }^{18} \mathrm{~F}-\mathrm{AV}-45$ PET images for two representative subjects. No artifacts or obvious misregistration can be observed (QC\#1).
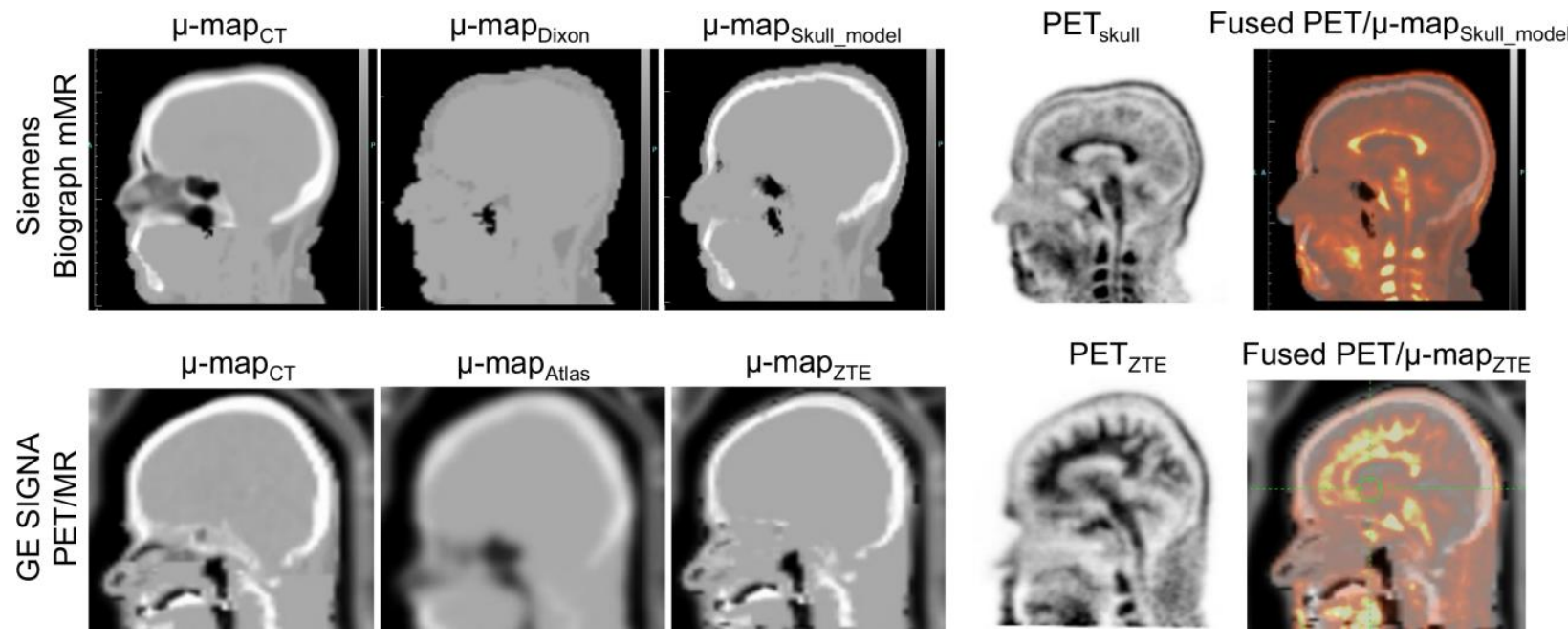
Figure 2: Cumulative voxel-wise relative differences between the PET images obtained using the four attenuation map generation methods and those generated using the reference CT-based approach for two representative subjects (blue). The voxel-wise relative differences are below $10 \%$ in at least $90 \%$ of the voxels included in the brain masks for the Siemens skull model- and GE ZTE-based approaches (QC\#2). The percentage of voxels with an absolute relative difference smaller than $10 \%$ is indicated in each case. The green line represents the histogram of the pixelwise percent difference.
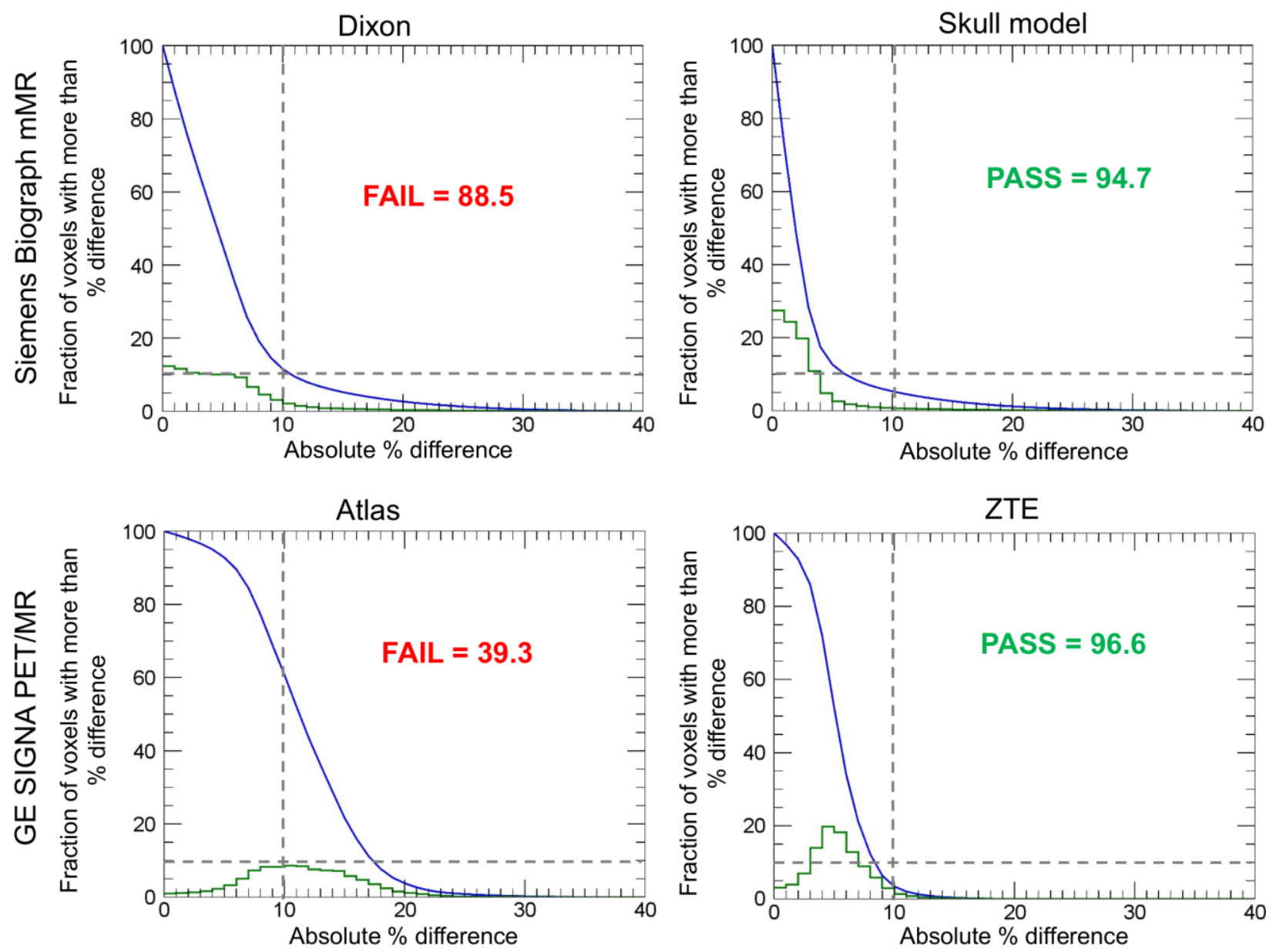
Figure 3: Bland-Altman plots of the absolute relative differences between the PET images obtained using the four attenuation map generation methods and those generated using the reference CTbased approach for the study-specific FreeSurfer-based regions of interest for two representative subjects. The average absolute percent differences are below $10 \%$ in all study-specific regions of interest for the Siemens skull model- and GE ZTE-based approaches (QC\#3).
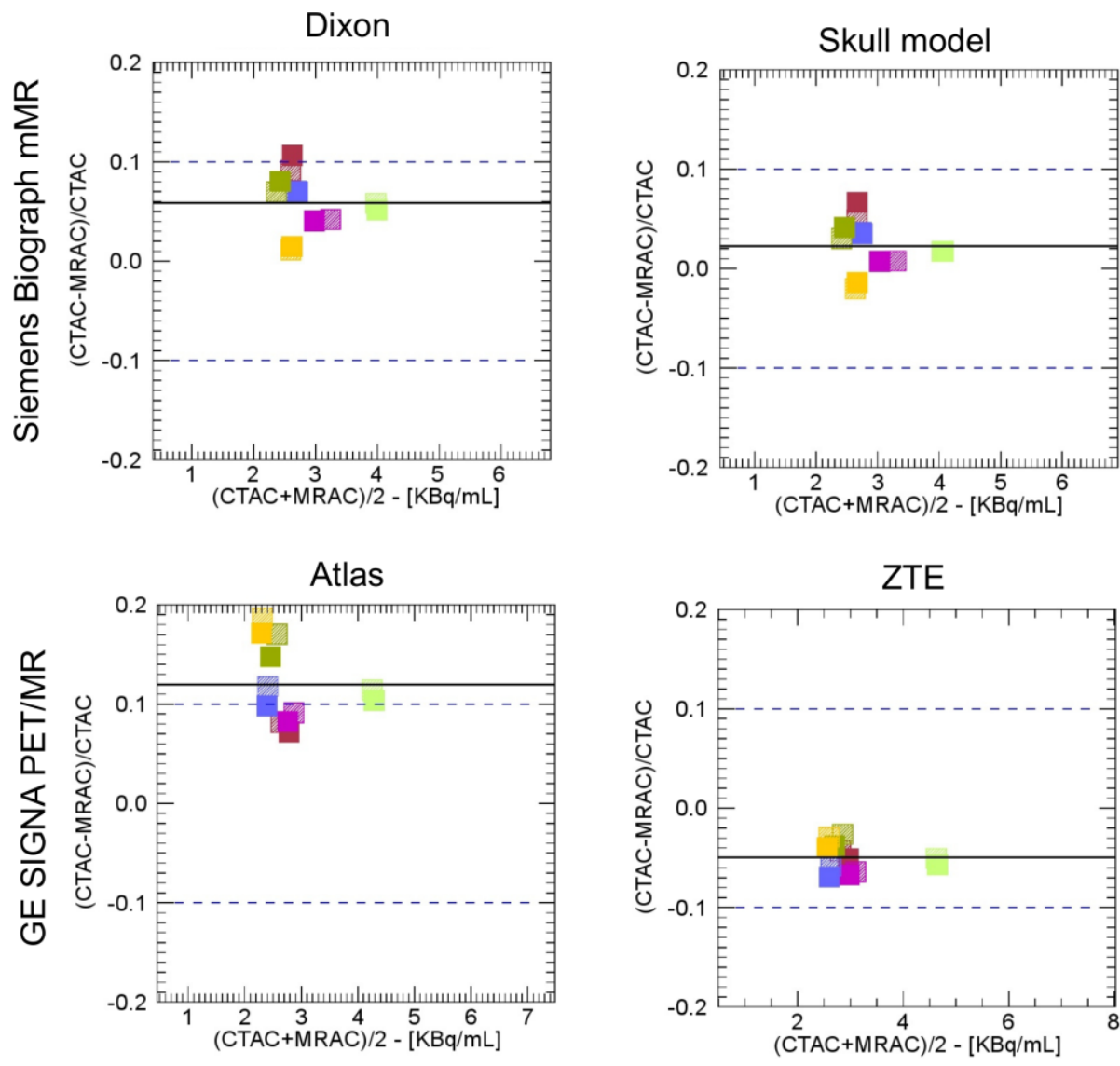

Left-frontal

Right-frontal

Left-cingulate

Right-cingulate

Left-parietal

Right-parietal

Left-lateral-temporal

Right-lateral-temporal

Left-white-matter

Right-white-matter

Left-cerebellar-cortex

Right-cerebellar-cortex 
Figure 4: Cumulative voxel-wise relative differences between the PET images obtained using the MR-based methods with respect to the CT-based approach for all 10 subjects analyzed. The solid curve represents the mean across the 5 subjects for each method. The voxel-wise relative differences are below $10 \%$ in at least $90 \%$ of the voxels included in the brain masks for all the subjects when using the Siemens skull model- and GE ZTE-based approaches.
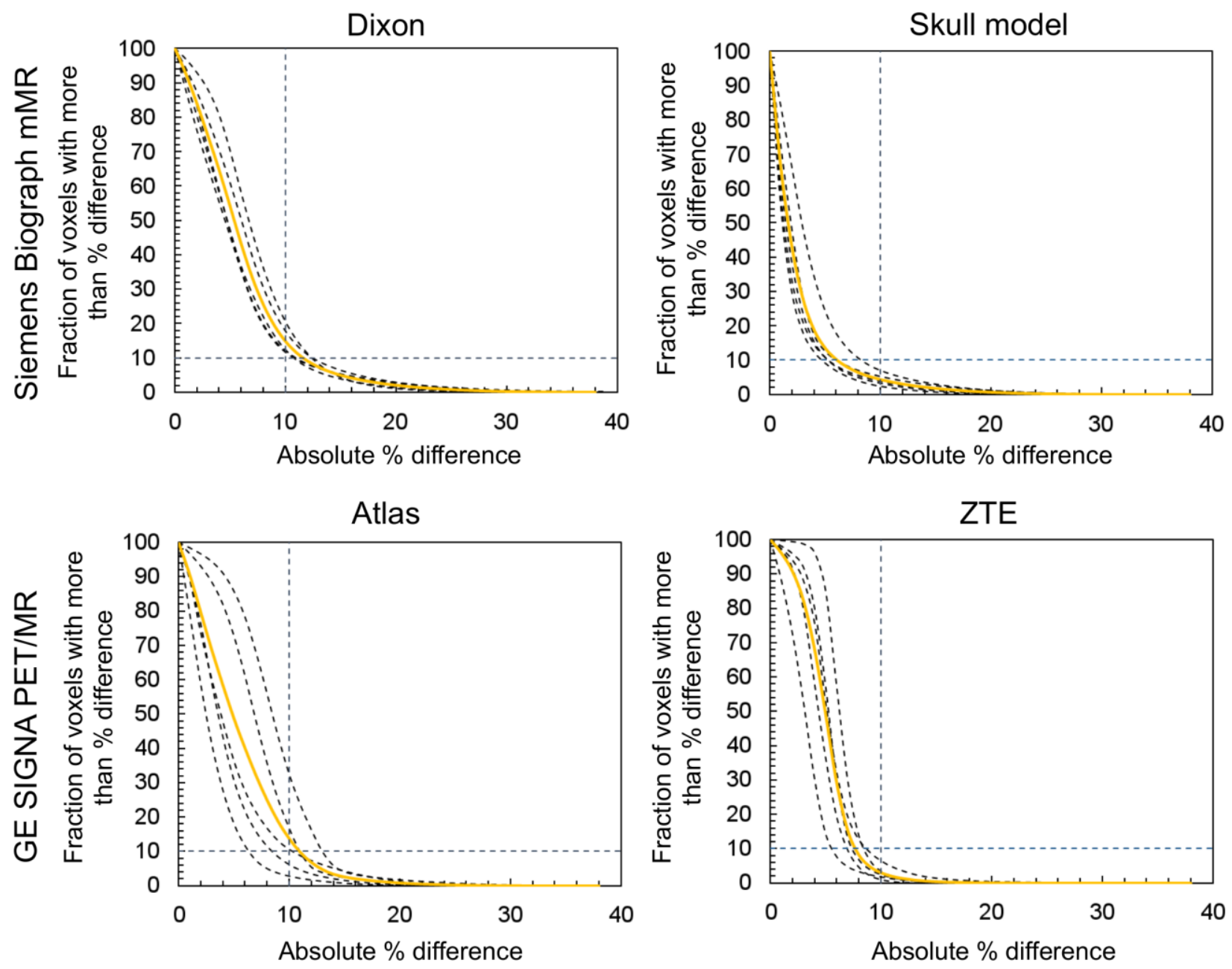


\section{Graphical Abstract}

Attenuation correction evaluation for brain imaging studies

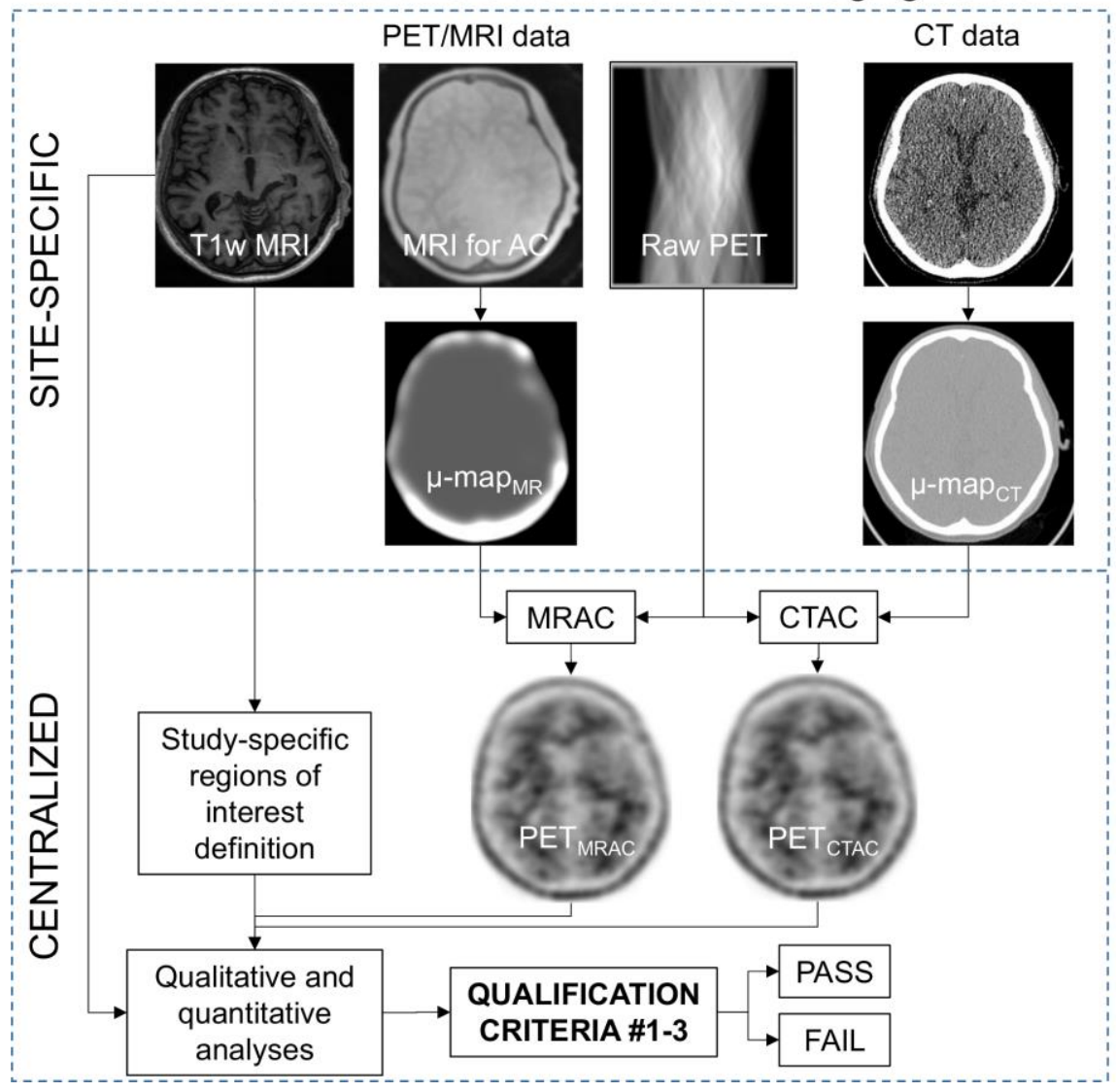

Separate evaluation of other effects relevant for PET data quantification

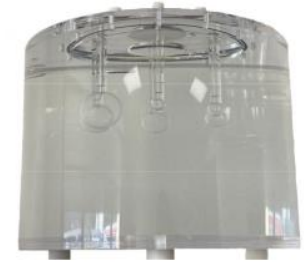

PET/CT phantom

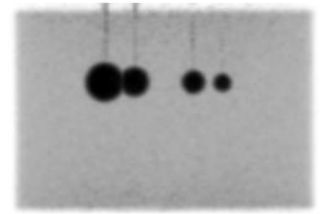

PET images (obtained using CTAC)

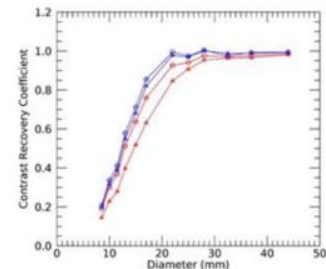

Recovery curves Study specific 


\section{Supplemental Methods}

\section{Institution 1}

Human subjects: Patients with mild cognitive impairment without a diagnosis of Alzheimer's disease ( 4 males, 1 female; average age of $73.4 \pm 10.7$ years) were enrolled for PET/CT followed by same-day PET/MRI studies.

CT data acquisition: Non-contrast studies were performed using the following acquisition parameters: voltage: $120 \mathrm{kVp}$, tube current: $20 \mathrm{~mA}$, slice thickness: $3.75 \mathrm{~mm}$.

\section{MRI data acquisition:}

1) Three-dimensional gradient echo $\mathrm{T} 1(\mathrm{BRAVO})$ : $\mathrm{TR} / \mathrm{TE}=7.9 / 3.0 \mathrm{msec}, \mathrm{TI}=450 \mathrm{msec}$, flip angle $=12^{\circ}$, matrix size $=256 \times 256, \mathrm{FOV}=25 \times 25 \mathrm{~cm}$, and slice thickness $=1 \mathrm{~mm}$, which results in a voxel size of $1.0 \times 1.0 \times 1.0 \mathrm{~mm}$.

2) Axial MR attenuation correction acquisition: $\mathrm{TE} 1 / \mathrm{TE} 2 / \mathrm{TR}=1.2 / 2.3 / 5.2 \mathrm{msec}$, flip angle $=$ $5^{\circ}$, matrix size $=256 \times 128$, slice thickness $=5.2 \mathrm{~mm}, \mathrm{NEX}=0.7$.

3) Zero echo time (ZTE): TR/TE $=404 / 0$, flip angle $=1^{\circ}$, matrix size $=110 \times 110, \mathrm{FOV}=25$ $\mathrm{x} 25 \mathrm{~cm}$, slice thickness $=2.4 \mathrm{~mm}, \mathrm{NEX}=4$, acquisition time $=21$ seconds.

PET data acquisition: ${ }^{18} \mathrm{~F}-$ florbetapir (Amyvid, AV-45, Eli Lilly) was administered with an average dose of $344.84 \pm 51.8 \mathrm{MBq}(9.32 \pm 1.4 \mathrm{mCi})$. The average delay between injection and imaging was $108.4 \pm 12.5$ minutes. Emission data were acquired for 15 minutes. The images were reconstructed with the vendor-provided time-of-flight, OSEM algorithm with 2 iterations and 28 subsets. A matrix size of $256 \times 256$ was used.

\section{Institution 2}

Human subjects: Patients with mild cognitive impairment without a diagnosis of Alzheimer's disease ( 2 males, 3 females; average age of $75.8 \pm 5.7$ years) were enrolled for PET/CT (Siemens Biograph $\mathrm{mCT}$ ) and PET/MRI. PET/MR scans were acquired $7.6 \pm 31.2$ days (range -17 to 62 days) after PET/CT scans. 
CT data acquisition: Non-contrast studies were performed using the following acquisition parameters: voltage: $120 \mathrm{kVp}$, tube current: $25 \mathrm{~mA}, 19.2 \mathrm{~mm}$ collimation, slice thickness: $3.0 \mathrm{~mm}$.

MRI data acquisition:

1) Sagittal three-dimensional Magnetization Prepared Rapid Gradient Echo (MPRAGE): $\mathrm{TR} / \mathrm{TE}=2300 / 2.95 \mathrm{msec}, \mathrm{TI}=900 \mathrm{msec}$, flip angle $=9^{\circ}$, matrix size $=240 \times 256, \mathrm{FOV}=$ $25.3 \times 27 \mathrm{~cm}$, and slice thickness $=1.2 \mathrm{~mm}$, which results in a voxel size of $1.0547 \times 1.0547 \times 1.2 \mathrm{~mm}$.

2) Coronal MR attenuation correction acquisition (DIXON): TE1/TE2/TR = 1.23/2.46/3.56 msec, flip angle $=10^{\circ}$, matrix size $=192 \times 126$, slice thickness $=3.12 \mathrm{~mm}$.

3) Coronal MR attenuation correction acquisition (CAIPIRINHA): TE1/TE2/TR = $1.28 / 2.51 / 4.14 \mathrm{msec}$, flip angle $=10^{\circ}$, matrix size $=384 \times 204$, slice thickness $=2.02 \mathrm{~mm}$.

PET data acquisition: ${ }^{18} \mathrm{~F}-$ florbetapir (Amyvid, AV-45, Eli Lilly) was administered with an average dose of $361 \pm 27.6 \mathrm{MBq}(9.76 \pm 0.75 \mathrm{mCi})$. The average delay between injection and imaging was 50 minutes. Emission data were acquired for 15 minutes. Images were reconstructed with the vendor-provided, 3D-OSEM algorithm with 3 iterations and 24 subsets, 4mm postreconstruction Gaussian filter size on $256 \times 256$ matrix size and $2.03 \mathrm{~m}$ slice thickness. 
Supplemental Figure 1: Attenuation maps, corresponding PET images, fused images and voxelwise percent difference maps for a representative subject scanned on the Siemens Biograph mMR.

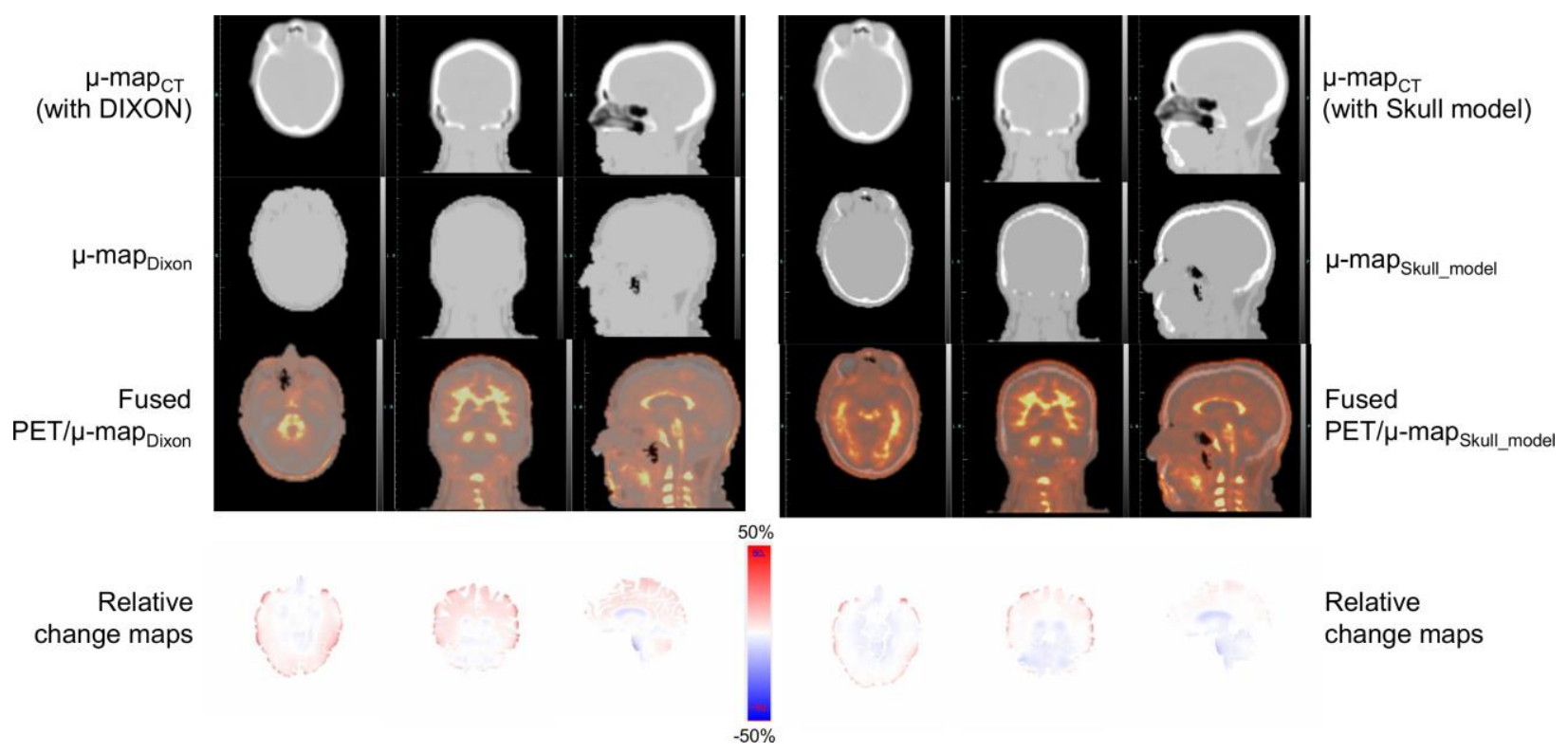


Supplemental Figure 2: Attenuation maps, corresponding PET images, fused images and voxelwise percent difference maps for a representative subject scanned on the GE Signa PET/MRI.

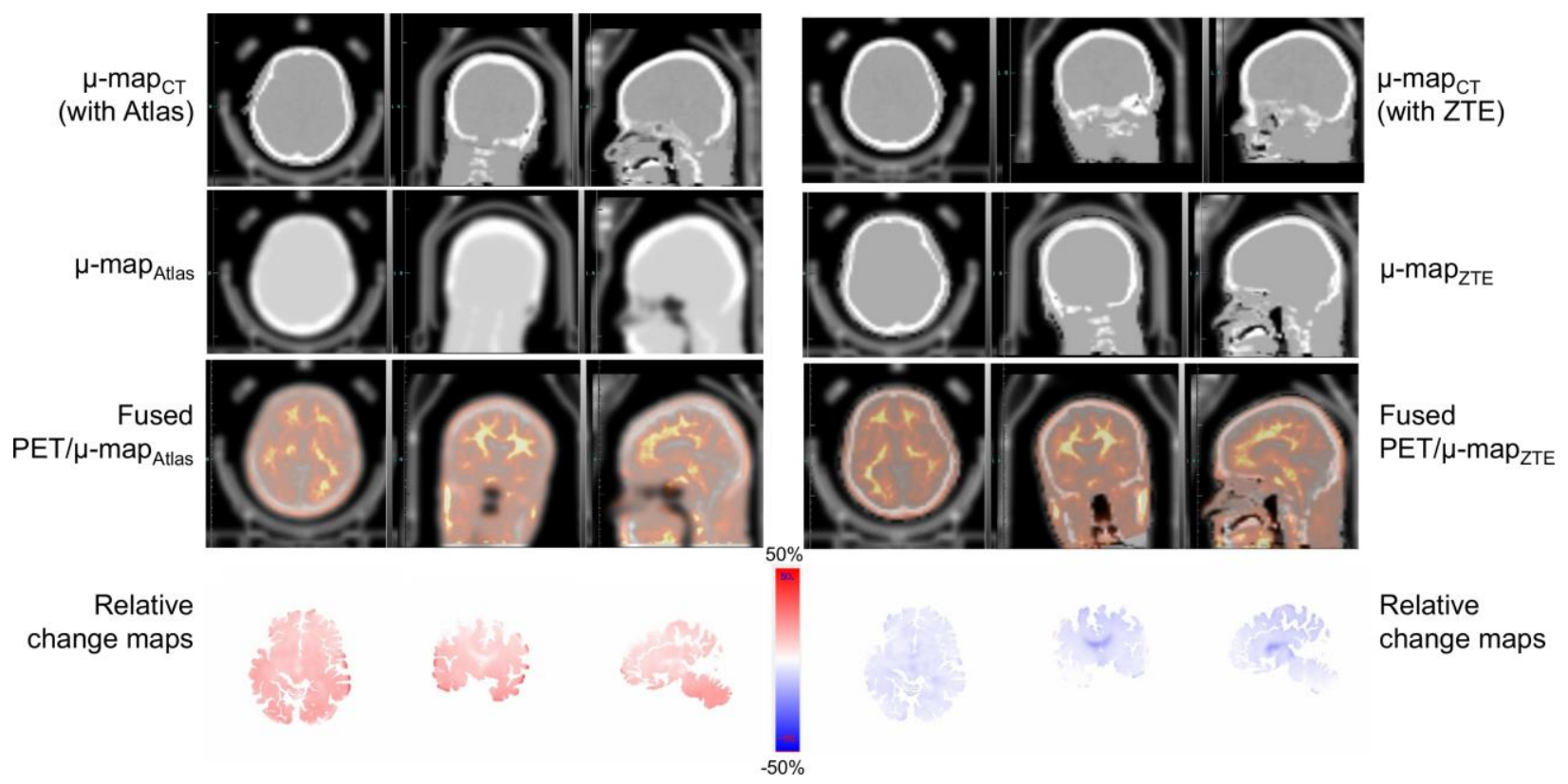


Supplemental Table 1: Percentage of voxels with an absolute relative difference smaller than $10 \%$ between the PET images obtained using the four attenuation map generation methods and those generated using the reference CT-based approach for all 10 subjects included in the analysis. Values greater than $90 \%$ are needed to satisfy QC\#2.

\begin{tabular}{|c|c|c|c|}
\hline \multicolumn{2}{|c|}{ Siemens Biograph mMR } & \multicolumn{3}{|c|}{ GE SIGNA PET/MR } \\
\hline DIXON & Skull Model & ATLAS & ZTE \\
\hline 88.5 & 94.7 & 93.8 & 96.5 \\
\hline 82.5 & 92.9 & 67.4 & 97.5 \\
\hline 79.5 & 97.5 & 39.3 & 96.6 \\
\hline 87.8 & 96.1 & 89.3 & 98.9 \\
\hline 87.7 & 96.7 & 97.1 & 98.1 \\
\hline
\end{tabular}

